\title{
Drug user dynamics: A compartmental model of drug users for scenario analyses
}

\author{
Mario Santoro ${ }^{1}$, Livio Triolo ${ }^{1}$, \& Carla Rossi $^{2}$ \\ ${ }^{1}$ Mathematics Department, University of Rome Tor Vergata, Rome, Italy and ${ }^{2}$ Department of System Medicine, \\ University of Rome Tor Vergata, Rome, Italy
}

\begin{abstract}
A six-typology compartment model of trends in the use of illicit drugs in Italy is developed to evaluate policies and interventions through scenario analyses. Inside the big compartments of just-cannabis users and multiple drug users, three subpopulations are taken into account: the occasional, regular and intensive user. Moreover, compartments for health care, assisted persons together with a constant source (susceptible) are added; removed people (i.e. those who leave drugs for any reason) are put in a compartment which does not participate in the dynamics. Flows from the susceptible reservoir among the drug-user compartments and to the removed or the health care compartments are studied in a deterministic linear ODE framework, where parameters are estimated using Italian data. This system shows an evolution towards a steady state, the speed of convergence being dependent on parameters in a fairly visible way. This model represents a theoretical development in drug policy analysis, as it shows the relevance of flux parameters, which are in principle subject to modifications due to institutional efforts, together with a quantitative evaluation of their role in the evolution of the whole system.
\end{abstract}

\section{INTRODUCTION}

The drugs problem and its consequences represent a complex field to investigate and to monitor. For policy makers, it is crucial to understand and 'measure' drug use and how it responds to drug control interventions in order to adopt evidence-based policies. In public health, as well as in other policy fields, the concept of indicators has been developed for data collection at large scale or when direct measurement is not possible for other reasons within the scope of monitoring.
Rather than aiming at exact estimates of prevalence and incidence of health problems, for example, indicators may provide indirect information, e.g. by giving insight in trends or phenomena associated with problems such as risk factors or consequences.

For drug epidemiology and monitoring at macro level (national and international), dynamic modelling can be a very powerful complementary tool to indicators and direct data analysis (Wiessing, Hartnoll, \& Rossi, 2001). Instead of the usual inductive or empirical method of data collection and interpretation, it is nearer to a deductive approach, where new insights follow from theory.

Dynamic models can help in understanding a phenomenon. If the model is assumed to describe a real-life process with sufficient accuracy, one can study the behaviour of this process under different circumstances, by varying parameter values and seeing the variation in outcomes. This semi-experimental situation is called 'what if' scenario analysis. Modelling can, thus, provide a tool to simulate experiments that are not possible in real life due to practical or ethical reasons (Levy, Chaloupka, Gitchell, Mendez, \& Warner, 2002; Levy \& Friend, 2002; Rossi, 2002). The importance of models to complement epidemiological data is put into evidence by considering the following:

(1) Prevalence can be estimated from epidemiological data while incidence is to be evaluated, by using some kind of modelling approach (back-calculation models, dynamic models, etc., see Scalia Tomba, Rossi, Taylor, Klempova, \& Wiessing, 2008, for details).

(2) Epidemiological data can just describe the phenomenon retrospectively, whereas models are essentially used to forecast possible future developments. 
Some modellers incorporate as many parameters in their models as possible, but sometimes, it is better to work with simple models that can easily be understood. Simple models that succeed in giving new insights are often more influential. Some models of both kinds have been developed in the decades since the pioneering work of Mackintosh and Stewart (1979) to mirror problem drug use epidemics (mostly heroin or injecting drug use epidemics) and consequences (Behrens, Caulkins, Tragler, \& Feichtinger, 2000; Behrens, Caulkins, Tragler, Haunschmied, \& Feichtinger, 1999; Billard \& Dayananda, 1993; Caulkins, Behrens, Knoll, Tragler, \& Zuba, 2004; Homer, 1993; Rossi, 2001, 2004).

A fundamental question resides in the possibility to reliably estimate parameters of the model or, at least, their order of magnitude. Considerations about the possibility of parameter estimation suggest to design models that are simple enough but not too simple, and that are data driven. This seems to be the approach for models recently developed to mirror particular situations worldwide, taking into account recent trends in drug use that do not allow using single substance models any more (Caulkins, Dietze, \& Ritter, 2007).

In the present contribution, one such model is presented and used to mirror the drug users' dynamics in recent years, perform scenario analyses and forecast future trends. The model is data driven and adapted to the present Italian situation. Minor modifications can be introduced to adapt the model for use in other countries. The choice of the structure of the model and of the compartments derives from the knowledge of recent trends in drug consumption in Italy, as widely described in the 'Data sources and parameter estimation' section. Drug consumption, in fact, shows higher and higher proportions of poly-use and it is not possible to distinguish patterns linked to a single substance except for cannabis. As a matter of fact, a specific subpopulation of 'pure' cannabis users can be considered separately from the other subpopulations of users, but it is not feasible to stratify the population with respect to the main substance of use nor the poly-drug users with respect to specific combinations of substances. Thus, the model is based on a series of compartments of pure cannabis users and a series of compartments for all the other users, including either single substance users or poly-drug users. It is possible to take into account frequency of use that is defined according to information coming from the surveys that are described in the 'Data sources and parameter estimation' section.

The model is a deterministic model representing a situation involving a large number of agents, so that a mean-field description (macro diffusion) represents the system in a satisfactory way. Should the number of involved people be small, a stochastic approach would be more appropriate. In this case, the description of the process should be based on the probabilities of contacts and of different possible behaviours of individuals involved in phenomenon (micro-level) and the outputs would be expressed in terms of probabilities of possible equilibrium values for the different subpopulations. As the size of the subpopulations considered in this article is big enough, then a macro-level approach is more appropriate.

In 'The model' section, we introduce the model and its mathematical aspects; in 'Parameters, basic scenario and unknown parameters evaluation' section, we represent and discuss different solutions; in the 'Different scenarios and possible interventions' section, we show different scenarios arising through suitable changes of parameters, in view of suggesting policies for getting better long-time results. The final section 'Data sources and parameter estimation' is devoted to a deep discussion of data sources and parameter estimation.

\section{THE MODEL}

Based on monitoring drug use in young people and in problem drug users in Italy, the following dynamic compartmental model of differential type is proposed. The compartments are defined by frequency of use and assortment of drugs, and the main issue is to extract from the estimated rates of change among different compartments the evolution of patterns of drug use. Two large compartments are first introduced, one corresponding to only-cannabis users $(C)$ and the other one to poly-drug users or users of other substances $(A)$. Each of these is further divided in three subcompartments, corresponding to consumption frequencies: occasionals (o) $<5$ times per month, regulars (r) 6-19 times per month and intensive (i) 20 or more times per month. This leads to six compartments: $C_{\mathrm{o}}, C_{\mathrm{r}}, C_{\mathrm{i}}, A_{\mathrm{o}}$, $A_{\mathrm{r}}$ and $A_{\mathrm{i}}$. The sizes of these subpopulations correspond to six dynamical variables, which enter the evolution equations. It is convenient to introduce another compartment $(H)$ which collects all people exiting from the intensive drug compartments for therapeutic treatment or social rehabilitation; moreover, there are exit fluxes to a removed users compartment that does not appear explicitly in the system: it contains, for instance, people who stop using drugs without entering into a health or social care process or die.

The source compartment $\left(S_{0}\right)$ represents susceptible people in the age segment $15-32$ years, and changes its composition at much a slower rate with respect to the drug compartments, so that it can be kept constant.

The changes in the sizes of compartments are described by a system of linear differential equations, where the compartment $S_{0}$ of susceptible subjects acts as a source, and the transitions among different compartments are described by the flux parameters which are estimated by statistical data (see 'Data sources and parameter estimation' section), and form the matrix of the coefficients. 


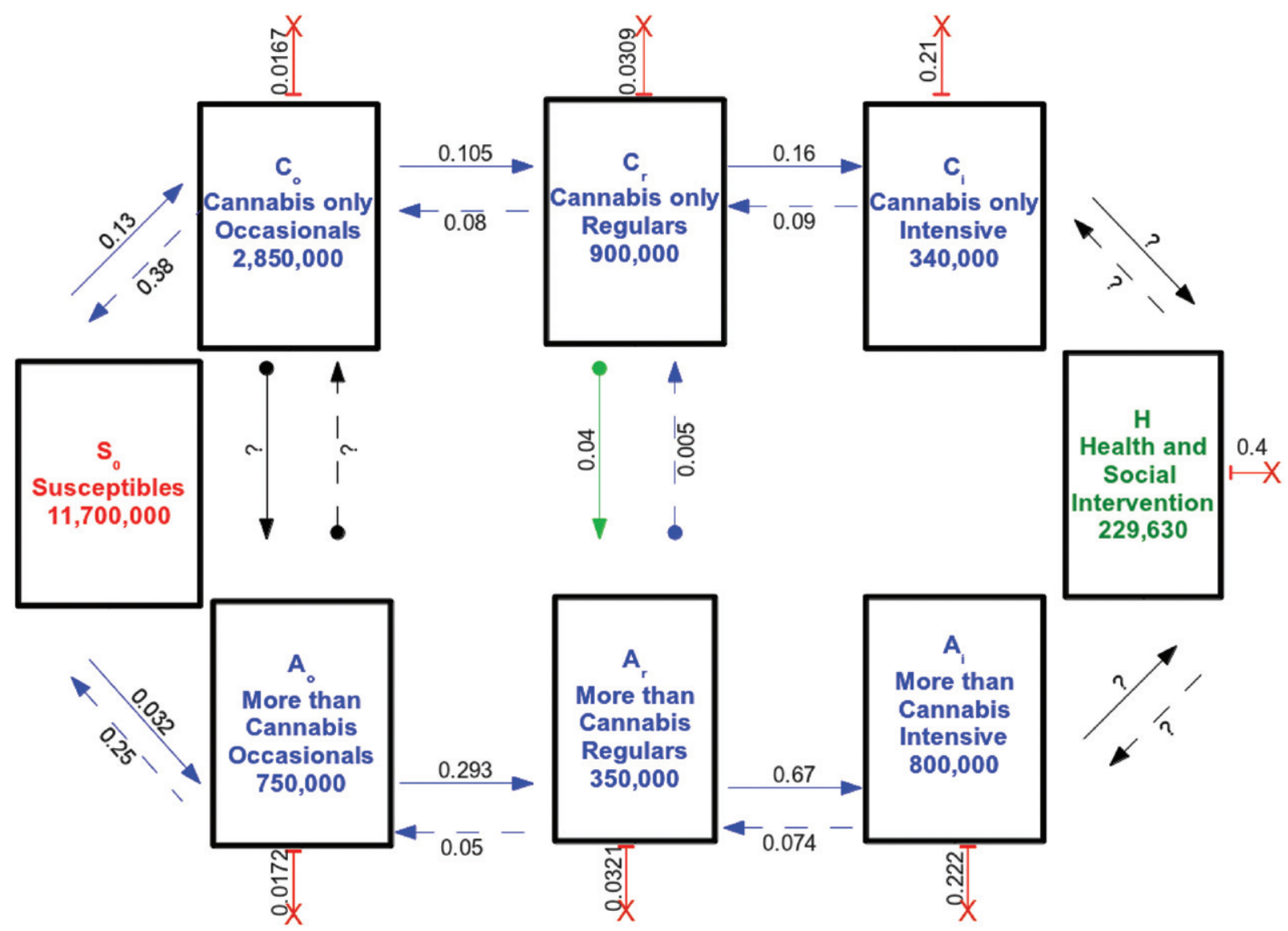

Figure 1. Block scheme of the model with initial estimates of parameters and populations of the compartments.

The model is graphically represented in the block scheme, as shown in Figure 1, where the values of the parameter and the sizes of the compartments are the initial estimates obtained in 'Data sources and parameter estimation' section. The arrows represent directions of flows between two compartments.

The mathematical description is the following linear differential system; the system is nonhomogeneous, due to the source term, and the dot on a symbol represents the time derivative of the associated quantity.

$$
\begin{aligned}
\dot{C}_{\mathrm{o}} & =\Lambda_{C_{\mathrm{o}}} S_{0}+\beta_{\mathrm{o}} A_{\mathrm{o}}+\gamma_{\mathrm{ro}} C_{\mathrm{r}}-C_{\mathrm{o}}\left(\delta_{\mathrm{o}}+\gamma_{\mathrm{ro}}+\mu_{C_{\mathrm{o}}}+d_{C_{\mathrm{o}}}\right) \\
\dot{A}_{\mathrm{o}} & =\Lambda_{A_{\mathrm{o}}} S_{0}+\delta_{\mathrm{o}} C_{\mathrm{o}}+\alpha_{\mathrm{ro}} A_{\mathrm{r}}-A_{\mathrm{o}}\left(\beta_{\mathrm{o}}+\alpha_{\mathrm{or}}+\mu_{A_{\mathrm{o}}}+d_{A_{\mathrm{o}}}\right) \\
\dot{C}_{\mathrm{r}} & =\beta_{\mathrm{r}} A_{\mathrm{r}}+\gamma_{\mathrm{or}} C_{\mathrm{o}}+\gamma_{\mathrm{ir}} C_{\mathrm{i}}-C_{\mathrm{r}}\left(\delta_{\mathrm{r}}+\gamma_{\mathrm{ro}}+\gamma_{\mathrm{ri}}+d_{C_{\mathrm{r}}}\right) \\
\dot{A}_{\mathrm{r}} & =\delta_{\mathrm{r}} C_{\mathrm{r}}+\alpha_{\mathrm{or}} A_{\mathrm{o}}+\alpha_{\mathrm{ir}} A_{\mathrm{i}}-A_{\mathrm{r}}\left(\beta_{\mathrm{r}}+\alpha_{\mathrm{ro}}+\alpha_{\mathrm{ri}}+d_{A_{\mathrm{r}}}\right) \\
\dot{C}_{\mathrm{i}} & =\gamma_{\mathrm{ri}} C_{\mathrm{r}}+v_{C_{\mathrm{i}}} H-C_{\mathrm{i}}\left(\gamma_{\mathrm{ir}}+\mu_{C_{\mathrm{i}}}+d_{C_{\mathrm{i}}}\right) \\
\dot{A}_{\mathrm{i}} & =\alpha_{\mathrm{ri}} A_{\mathrm{r}}+v_{A_{\mathrm{i}}} H-A_{\mathrm{i}}\left(\alpha_{\mathrm{ir}}+\mu_{A_{\mathrm{i}}}+d_{A_{\mathrm{i}}}\right) \\
\dot{H} & =\mu_{C_{\mathrm{i}}} C_{\mathrm{i}}+\mu_{A_{\mathrm{i}}} A_{\mathrm{i}}-H\left(v_{C_{\mathrm{i}}}+v_{A_{\mathrm{i}}}+d_{H}\right)
\end{aligned}
$$

The solution, for any initial state, shows a convergence to a stationary state solution of the associated linear stationary problem. This solution is globally asymptotically stable as all eigenvalues of the matrix of the coefficients are negative.

\section{PARAMETERS, BASIC SCENARIO AND UNKNOWN PARAMETERS EVALUATION}

In order to calculate the numerical solution of the system, the Runge-Kutta fourth-order method via the lsoda package in $\mathrm{R}$ software has been used. Initial conditions are: occasionals $(C$ and $A$ ) equal to 100 and other subpopulations 0 . This initial condition is fairly distant from the steady solution which represents the actual situation in Italy; this describes the breadth of the basin of attraction of the steady state.

All dynamical variables are represented as functions of time (stabilization is seen after approximately 10 years) and the system evolves to a steady state, as shown in Figure 2.

In order to obtain this solution, the parameters are refined, or evaluated for the unknown ones (question marks in Figure 1), with a sensitivity analysis using the following criteria:

(1) The already known parameters cannot change more than $25 \%$ from data sources evaluation.

(2) Also, the steady-state values from data sources cannot change more than $25 \%$.

(3) Twenty-five per cent is the maximal relative uncertainty of parameter evaluations from data sources. 


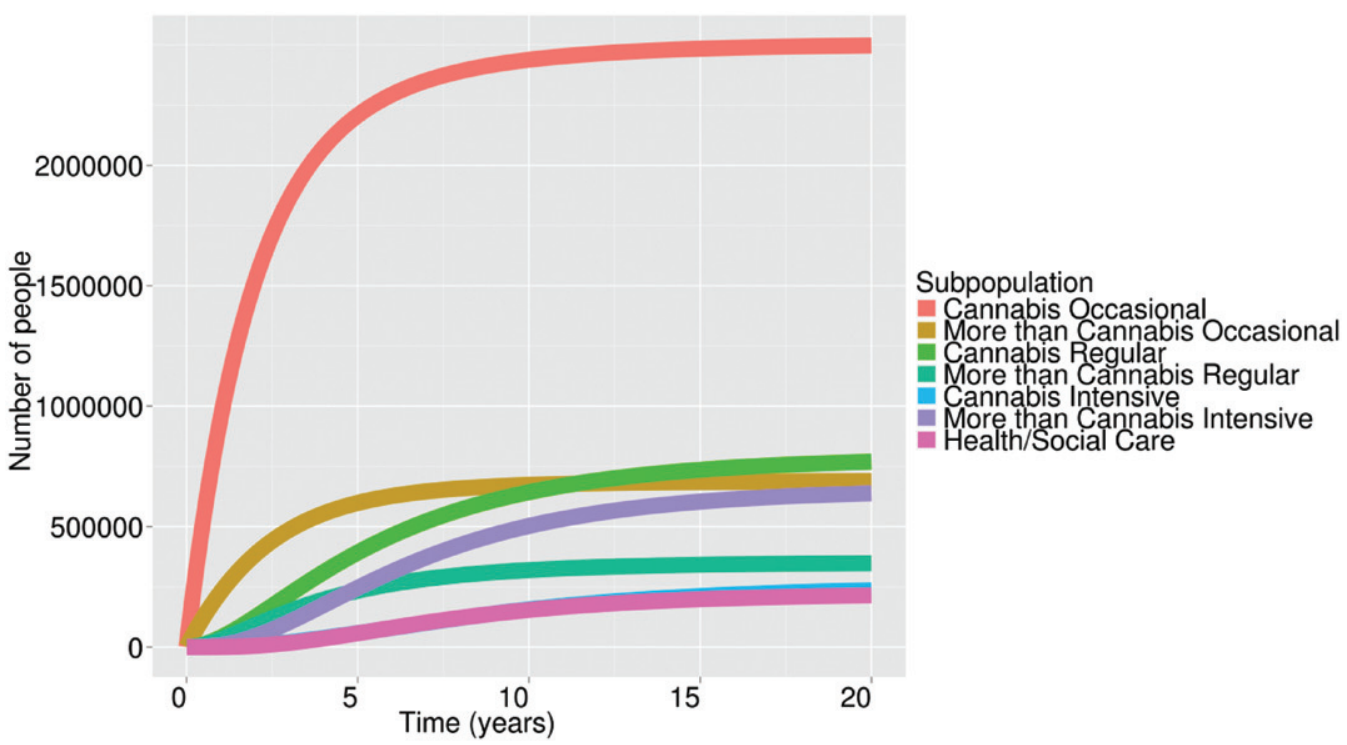

Figure 2. Time behaviuor of the populations of the various compartments in the basic scenario.

The adjusted parameter values are reported in the scheme of Figure 3.

\section{DIFFERENT SCENARIOS AND POSSIBLE INTERVENTIONS}

By modulating parameters, various policies can be analysed: by changing some flux parameters (rates of entrance from susceptibles, or backward fluxes from health/social care compartment, etc.), their role can be evaluated in fixing the long-run equilibrium values and the speed of convergence. In this way, one may appreciate the relevance of some rates with respect to others, and, in principle, conceive strategies in order to get better results. In the following, few simulations with reduced flow parameters and the relative graphs are shown.

(1) If the input flow decreases by $20 \%$ after 20 years since the beginning, the result is represented in Figure 4(a) and related per cent changes in the stationary values in Figure 4(b).

(2) Decreasing by $20 \%$ the occasional-to-regular flow, after 20 years since the beginning, the result is represented in Figure 5(a) and related per cent changes in the stationary values in Figure 5(b).

(3) Twenty per cent decrease for three flows: input, occasional-to-regular, health/social care-tointensive after 20 years since the beginning, produces the results in Figure 6(a) and related per cent changes in the stationary values in Figure 6(b).

Using this model, it was possible to integrate standard epidemiological analysis and forecasts. It was also possible to estimate some transition rates that could not be evaluated on the basis of available data (question marks in Figure 1).

\section{DATA SOURCES AND PARAMETER ESTIMATION}

Information for parameter estimation comes from various sources: demographic databases, administrative databases and surveys.

\section{Demographic databases}

Demographic parameters of the model, namely the size of the cohort that enters yearly in the age at risk for onset of drug use (susceptibles), i.e. the age classes 1532 , and the death rate for susceptibles and for occasional cannabis users are derived from the demographic databases of the National Statistical Institute available online (http://www.istat.it/it/popolazione).

\section{Administrative sources}

Administrative databases are used to estimate some parameters related to transitions between the drug user compartments and the therapy compartment and other transition rates. They are also used to estimate the prevalences in the compartments of the steady state. Several databases coming from various public agencies are available. In order to estimate the parameters of the model, the following ones have been used:

(1) The data set provided by the Ministry of the Interior (MI) of registrations for personal use of illegal substances. Individuals are unequivocally identified and recorded and may appear in the data set several times. Life histories can be followed during the years 2005-2010. The information about the main substance of use is always recorded as well as age, gender, geographical area, etc.

(2) The data set provided by a sample of public health care services where clients are classified by primary and secondary drug used; age, gender, age at first use and time lag between first drug use and first treatment entry (AKA latency period) are 


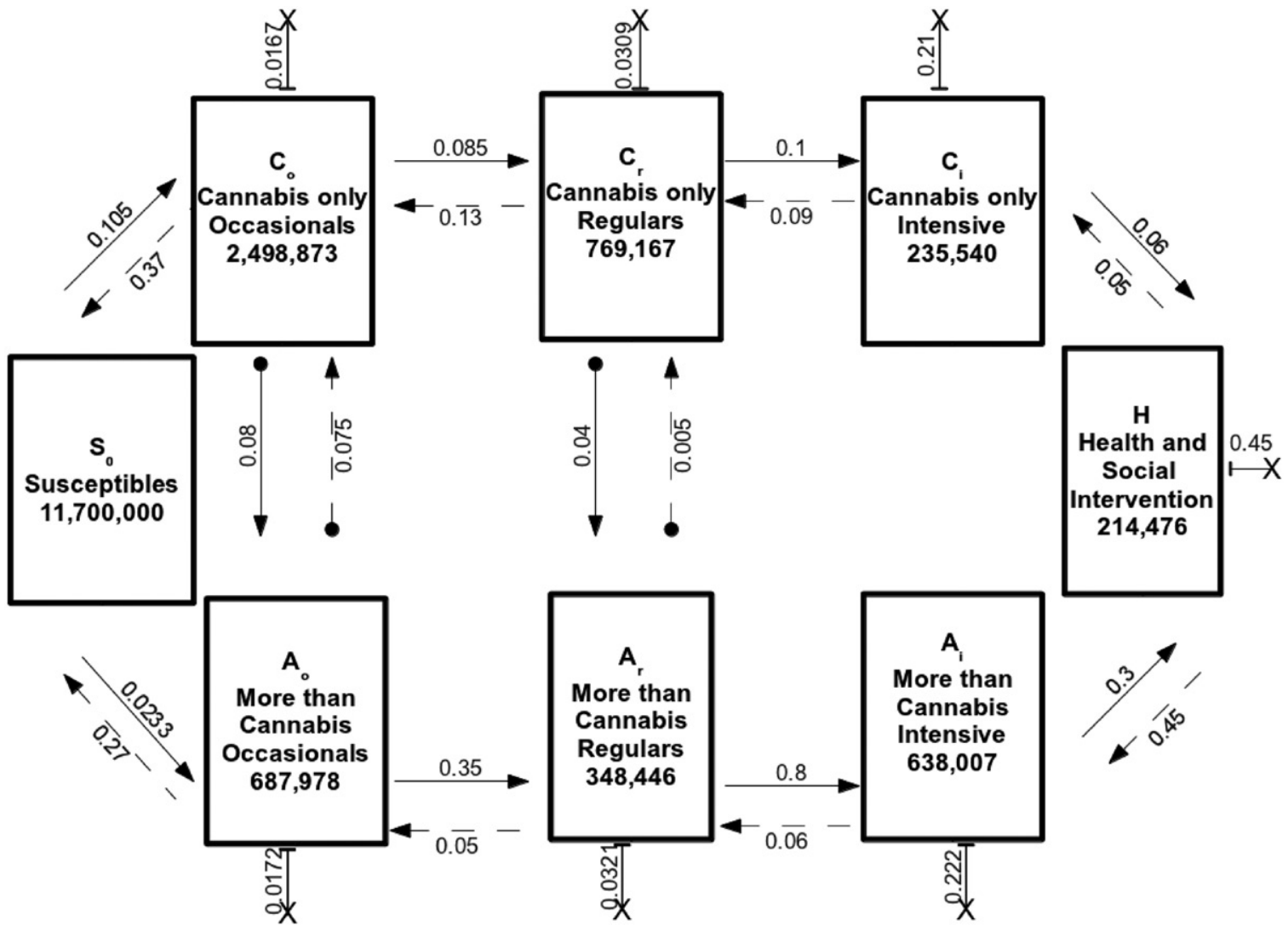

Figure 3. Block scheme of the model with final estimates of parameters and populations of the compartments obtained by simulation and sensitivity analysis.

also recorded along with pattern of drug use and route of administration; socio-demographic data are also available. The data set is available for the years 2009 and 2010.

(3) Aggregated data set provided by the Ministry of Health on clients assisted by public services where prevalence and incidence of drug treatment demand can be obtained. The data set is available for several years and, in particular, for the years 2005-2010.

(4) Data set of the subjects registered for dealing drugs. Individuals are unequivocally identified and recorded and may appear in the data set several times. Age, gender and geographical area are available. Life histories can be followed during the years 2005-2010.

\section{Data from surveys}

Several data sets from surveys are available for the years 2010, 2011 and 2012.

(1) The School Population Surveys SPS 2010 and SPS 2011, conducted in Italy by the University of Rome 'Tor Vergata' for the Anti-Drug Department of the Presidency of the Council of Ministers. The surveys were conducted in the period FebruaryMay in the years 2010 and 2011. The response rate was around $80 \%$ for both years (Dipartimento
Politiche Antidroga, 2010, 2011). An online questionnaire was utilized: about 34,000 valid records were collected in 2010 and about 33,000 in 2011. The questionnaires comprise all the questions of the ESPAD project (http://www.espad.org/) plus further ones regarding lifestyles and the drug market. The data sets include information on substances used, frequency of use (lifetime, last 12 months and last 30 days), poly-use, pricing, funding and other variables providing a significant amount of data to analyse the lifestyle of teenagers in their first encounter with licit and illicit drugs.

(2) The Survey in the Therapeutic Communities and in the Low Threshold Services conducted in 2010 in Italy by the University of Rome 'Tor Vergata' for the Anti-Drug Department of the Presidency of the Council of Ministers. About 1500 residents in therapeutic communities or clients of low threshold services filled a questionnaire with several questions about: social and demographic characteristics; drugs used, age of first use and heavy use of drugs; and drug correlated behaviour (reasons, places, prices, route of administration of drugs). The survey was repeated with a more complete questionnaire in 2012 in the framework of the project financed by the EU Commission New methodological tools for policy and programme evaluation by the Centre of Biostatistics and 
(a)
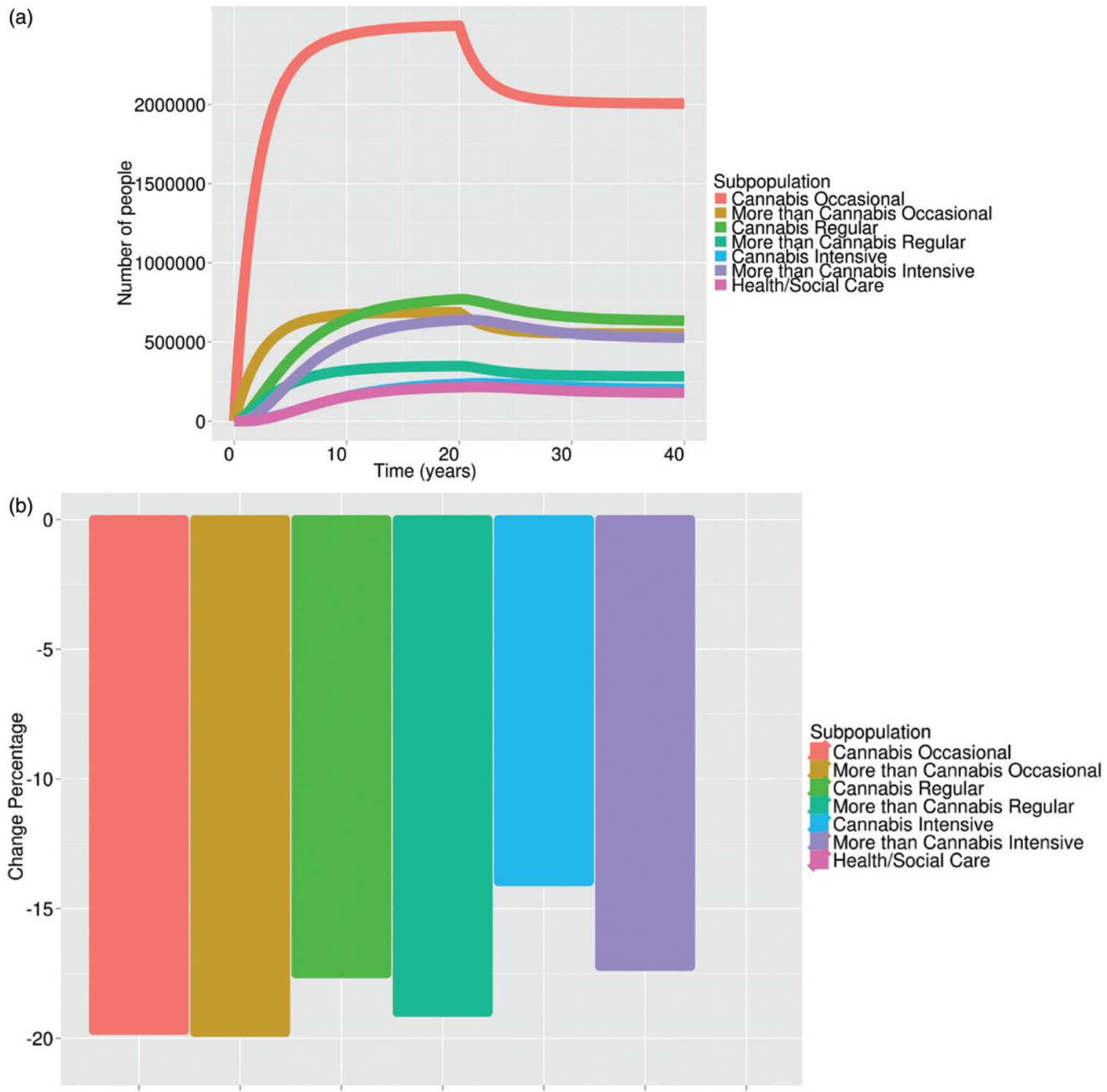

Figure 4. Time behaviuor of the populations of the various compartments (a) and related per cent changes in the stationary values (b) in scenario 1.

Bioinformatics of the University of Rome 'Tor Vergata'. Presently, 720 questionnaires are available from Italy.

(3) The online survey among occasional and regular users and the interviews to clients of Communities and Low Threshold Services conducted by the Centre of Biostatistics and Bioinformatics of the University of Rome 'Tor Vergata' in the framework of the EU project Study on the further analysis of the EU illicit drugs market and responses to it - responding to future challenges that allows for further information about lifestyles, drug use onset and drug users career.

Combining the information from all the data sources, it is possible to estimate several parameters of the model, or at least their order of magnitude to be refined by sensitivity analysis and the prevalences in the compartments at the steady state.

\section{Parameter estimation}

First, the prevalence at steady state is considered. For this purpose, it is necessary to assume that the present situation, with respect to the compartments of the model, is the steady state. This assumption is supported by observations and estimations of the main prevalence indicators and proxies showing that the population of users in the six compartments is fairly constant in the last 5 years. Whereas, the number and type of substances used in the three compartments ' $A$ ' is continuously changing (MI, estimate of the drug dealer population, surveys among clients of Communities and Low threshold Services, SPS 2010 and 2011). 
(a)
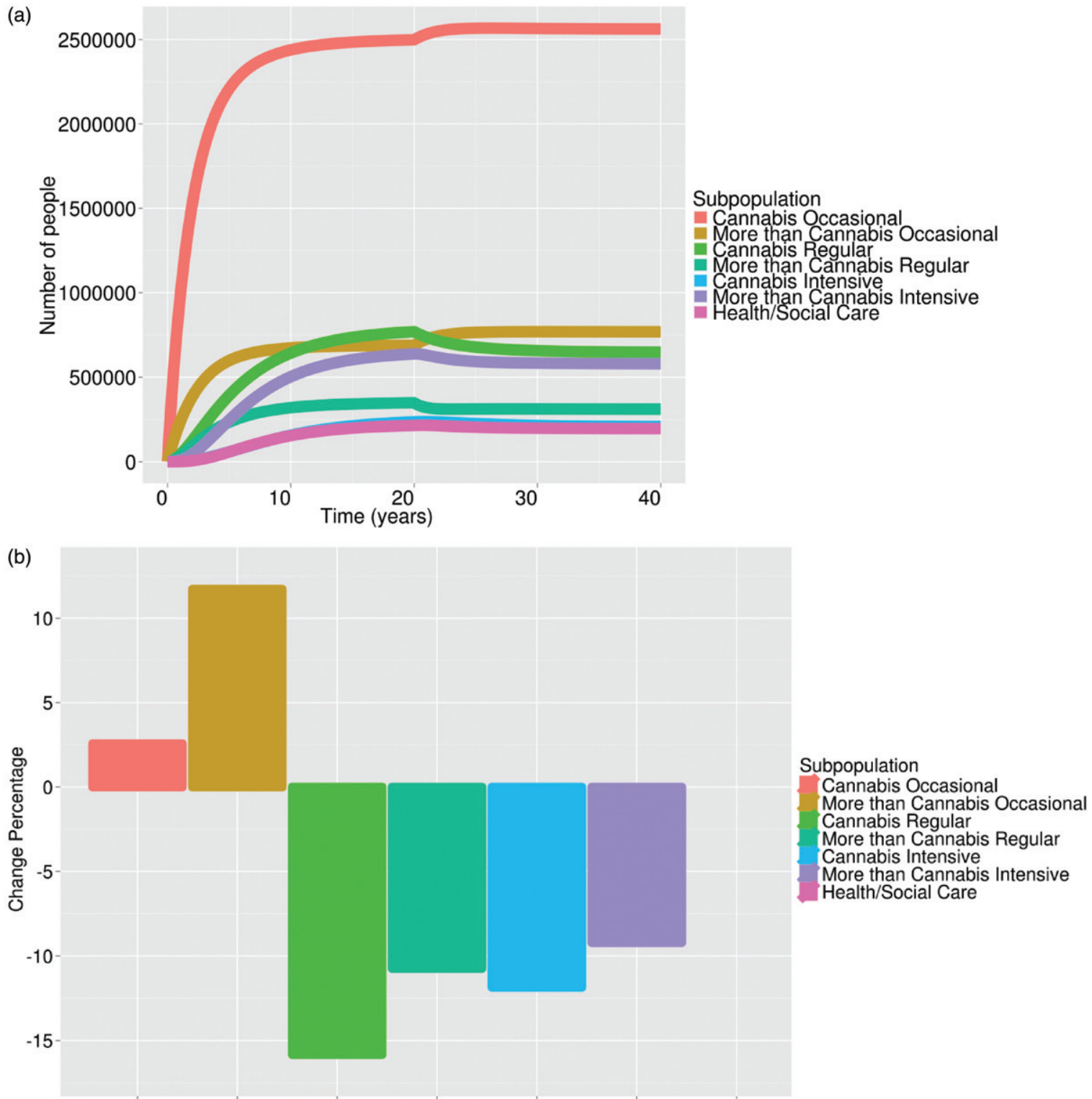

Figure 5. Time behaviuor of the populations of the various compartments (a) and related per cent changes in the stationary values (b) in scenario 2.

\section{Prevalence estimation of the total population of drug users and of the populations in the various compartments of use}

This point might be addressed using data from General Population Surveys. Unfortunately, in Italy, the response rate for this kind of surveys is very low in recent years $(<25 \%)$ and the estimates are not reliable; though it is possible to find an estimate by calibration from a robust estimate of the drug dealer population. This population has been recently estimated using Zelterman estimate from the database of subjects registered for dealing drugs (Rossi in Rey, Rossi, \& Zuliani, 2011). The estimates of the dealer population at national level are fairly constant during the period of interest. Using information from the literature, in particular from Bouchard and Tremblay (2005), about the ratio consumers/dealers for the various substances, it is possible to estimate the global population of drug users and poly-drug users by multiplying the estimated population of dealers by the typical ratios for the various substances. Table I provides the synthesis of the estimation.

A correction is needed for cannabis dealers, as there are some of them who are not at risk of being registered; using the same underestimation parameter as in Bouchard and Tremblay (2005) (14\%), the adjusted estimate of the active dealer population for cannabis is about 125,500 and for cannabis user population is about $4,000,000$.

This provides a total for the drug user and poly-user population of 5,750,000. It must be taken into account that the estimation made on the basis of the substances dealt and used produces some double counting as dealers can sell more than one substance $(20 \%$ of the 
(a)
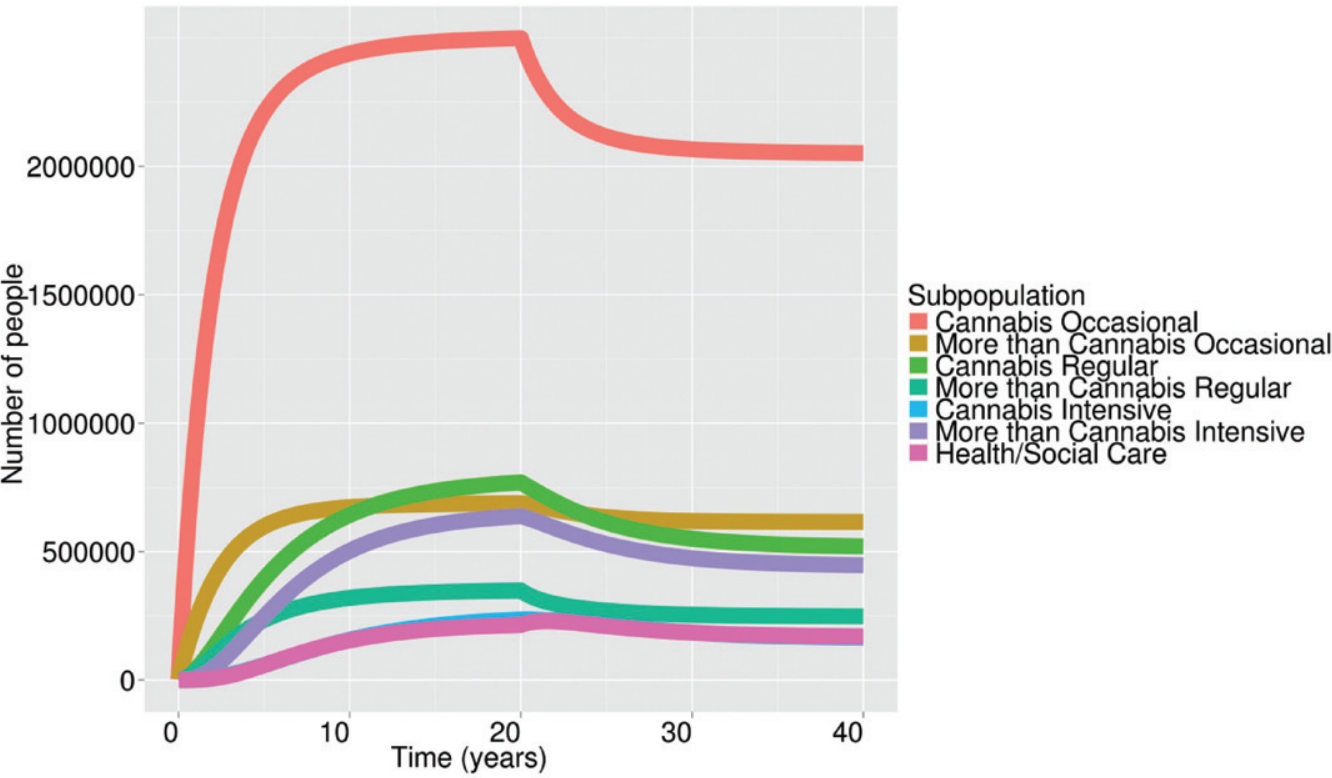

(b) 0 -

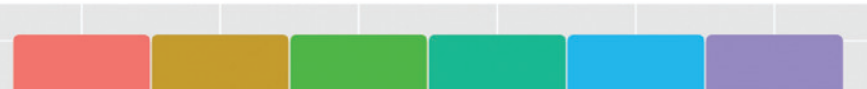

$-5$

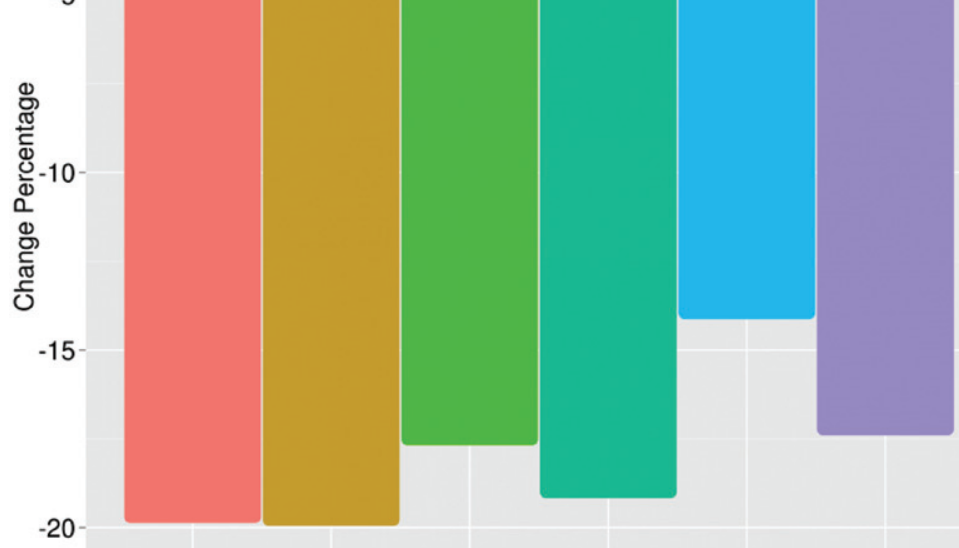

Figure 6. Time behaviuor of the populations of the various compartments (a) and related per cent changes in the stationary values (b) in scenario 3.

Table I. Estimated dealer and user populations (yearly average over the period 2005-2009).

\begin{tabular}{|c|c|c|c|c|}
\hline Substance & $\begin{array}{c}\text { Estimated } \\
\text { dealers }\end{array}$ & $\begin{array}{c}\text { Adjusted } \\
\text { active } \\
\text { dealers } \\
\text { (6 months of } \\
\text { activity per year) }\end{array}$ & $\begin{array}{c}\text { Customer-to-seller } \\
\text { ratio } \\
\text { (Bouchard \& } \\
\text { Tremblay, 2005) }\end{array}$ & $\begin{array}{l}\text { Estimated users } \\
\text { and poly-users }\end{array}$ \\
\hline Cannabis & 220,000 & 110,000 & 32 & $3,520,000$ \\
\hline Cocaine & 88,000 & 44,000 & 25 & $1,100,000$ \\
\hline Opiates & 72,000 & 36,000 & 16 & 500,000 \\
\hline Other & 20,000 & 10,000 & 15 & 150,000 \\
\hline Total & 400,000 & 200,000 & & $5,270,000$ \\
\hline
\end{tabular}


Table II. Estimates of consumers and poly-consumers (average over the period of interest).

\begin{tabular}{lccc}
\hline Substance & Consumers & Substance & Consumers \\
\hline Cannabis & $4,000,000$ & Cannabis & $4,000,000$ (double counting) \\
Cocaine & $1,100,000$ & Cocaine & $1,100,000$ (double counting) \\
Other (comprising opiates and those in therapy) & 850,000 & Other & 150,000 (double counting) \\
Total & $5,950,000$ & Opiates & 500,000 \\
Number of opiate users & 500,000 & Users in therapy & 200,000 \\
& & or in social services \\
\hline
\end{tabular}

seizures concern more than one substance) and polyusers are counted more than once.

The estimates of the users can be adjusted on the basis of other sources of information and, in particular, of the surveys that allow for estimating the coefficient of poly-use (Fabi et al. in Rey et al., 2011) for the various compartments of poly-users.

First, a double check of the estimates of the user population of cannabis and cocaine can be performed. These populations can be directly estimated on the basis of the data set of the registrations for personal use of drugs. The estimate of the population of cannabis users at risk of registration has been obtained by Mascioli and Rossi (2008) and is about 700,000. This estimate is an underestimate because only the covariate 'district' has been included and because the proportion of susceptibles at risk of registration is relatively small for cannabis users. The group at risk is mainly constituted by those users who consume in public settings. The proportion of this population can be estimated on the basis of the data coming from the online survey of 2012 for cannabis and cocaine users.

The estimate of the population at risk of registration is for the cannabis user about 700,000 and for the cocaine user about 170,000 (Zelterman estimate from MI data set). The proportion of cannabis users who consume in public settings is about $18 \%$ and of cocaine users is about $17 \%$. This allows for an adjustment of the estimate of the population of cannabis and cocaine users. The adjusted estimates are: about $3,900,000$ for the cannabis user population and about $1,000,000$ for the cocaine user population.

These estimates suffer from double counting due to poly-use, as with the estimates reported in Table I.

The similarity of the estimates obtained by a simple multiplier on the basis of the estimates of the dealer population and those obtained by applying the Zelterman estimator to the registrations for personal use is impressive.

The problem is now the correction of the double counting and the distribution of the users in the six compartments.

In Table II, the estimates are summarized.

In order to weight poly-use and intensity of use, information from surveys are valuable.

The distribution of consumers from SPS in the frequency of use compartments is: occasional $(60 \%)$,
Table III. Estimates of the prevalences in the six compartments of users (steady state).

\begin{tabular}{lrrrr}
\hline & \multicolumn{4}{c}{ Frequency of use } \\
\cline { 2 - 5 } Substance & Occasional & Regular & Intensive & Total \\
\hline Only cannabis & $2,500,000$ & 700,000 & 212,000 & $3,412,000$ \\
Other & 660,000 & 300,000 & 640,000 & $1,600,000$ \\
Total & $3,160,000$ & $1,000,000$ & 850,000 & $5,012,000$ \\
\hline
\end{tabular}

regular $(21 \%)$ and intensive $(19 \%)$. From the online survey and the survey in the Communities and Low Threshold Services, the analogous distributions are obtained for the intensity of use and can be combined providing a proxy of the distribution of intensity of use for the general population: occasional (53\%), regular (22\%) and intensive (25\%). Regular and intensive users are still affected by double counting for poly-use. This provides the following prevalences: occasional users $(3,160,000)$, regular users $(1,310,000)$ and intensive users $(1,500,000)$.

Pure cannabis users represent $79 \%$ of occasional users, $70 \%$ regular users and $25 \%$ intensive users.

The poly-use coefficients that must be applied to intensive and regular users to correct for double counting are 1.48 (intensive) and 1.21 (regular) (Fabi et al. in Rey et al., 2011; Fabi, Mammone, \& Rossi, 2012). Thus, the adjusted estimates of the consumer population in the three segments of frequency of use is: occasional users $=3,160,000, \quad$ regular users $=1,000,000$ and intensive users $=850,000$, users in therapy or in social service, about 200,000 (known and constant in the past decade).

By applying the information about the proportion of pure cannabis users to the three segments, the results of Table III are obtained. These must be considered rather conservative as any hypothesis for the estimates has been assumed at the lower level. However, the order of magnitude of the compartments can be consider fairly reliable and can be adjusted by sensitivity analysis.

Overall, pure cannabis consumers represent $68 \%$ of the consumer population.

Opiate represent over $30 \%$ of the consumer of substances 'other' than only cannabis. 
The rest is mostly represented by cocaine users and users of various combinations of substances, comprising cannabis.

\section{The prevalence in the susceptible compartment and the death rates}

The prevalence of the susceptibles is fixed at $11,700,000$ - that is the size of the resident population aged 15-32, the age class most involved in drug use initiation and is fairly constant in recent years in Italy. The death rate of the occasional users of only cannabis has been set equal to the death rate of the resident population in the same age classes. The death rates of the other compartments have been modulated according to the literature with respect to mortality of drug users (longitudinal studies) and the age classes mostly represented in the compartments. The values of the death parameters are: $d_{C_{\mathrm{o}}}=0.0167 ; d_{A_{\mathrm{o}}}=0.0172$; $d_{C_{\mathrm{r}}}=0.0309 ; \quad d_{A_{\mathrm{r}}}=0.321 ;$ and $d_{C_{\mathrm{i}}}=0.0310+0.179$ (definitely quit drug use); $d_{A_{\mathrm{i}}}=0.2222 ;$ and $d H=0.2222+0.179$ (definitely quit drug use).

\section{The forward and backward transition rates between susceptibles and occasional users}

The age at onset of drug use can be derived from the various surveys available (SPS 2010 and 2011, Communities and online survey on occasional and regular users). The same data sets provide the information on substance of first use.

Age at onset for cannabis, which is the first substance of use for about $80 \%$ of the users, is very regularly distributed in the various data sets with the mode at 15 , the median at 15.5 and the 95 th percentile between 19 and 20. This implies that most information about cannabis use onset can be obtained from SPS 2010 and 2011, with the assumption that individuals of the same age, who are not in school, have the same behaviour with respect to drug use initiation as school students (conservative assumption). From the online survey on occasional and regular users, the age distribution at the onset of regular use of cannabis can be obtained and shows the mode at 16 , the median at 18 and the 95th percentile between 23 and 24 . The comparison of the means allows to estimate a lag of about 2 years from first use to regular use for those who proceed. The results of all these analyses allows for an estimate of the yearly transition rate from susceptibles to occasional user of cannabis only ( $C_{\mathrm{o}}$ compartment) $\Lambda_{\mathrm{C}}=0.13$ and the yearly transition rate from susceptibles to occasional user of other drugs $\left(A_{\mathrm{o}}\right.$ compartment) $\Lambda_{\mathrm{A}}=0.032$.

From SPS 2010 and 2011, it is possible to evaluate the proportion of those who quit their use comparing the answers of the question about lifetime use, last 12 months use and last 30 days use either for only cannabis users or for users of other drugs. The transition rates are, respectively, $\mu A_{\mathrm{o}}=0.25$ and $\mu C_{\mathrm{o}}=0.38$, showing that the transitions (forward and backward) between susceptibles and occasional users are rather frequent.

\section{The forward and backward transition rates between occasional and regular users}

The transition rates between occasional and regular users can be estimated using the information from SPS 2010 and 2011. It is possible to set up a table with the last 12 months frequency of use and the last 30 days frequency of use. From the comparison, it is possible to estimate the transition rates between the compartments that result: $\gamma_{\mathrm{or}}=0.105 ; \gamma_{\mathrm{ro}}=0.08 ; \alpha_{\mathrm{or}}=0.283$; and $\alpha_{\text {ro }}=0.05$. The choice of estimating these parameters on the basis of the SPS 2010 and 2011 is rather conservative. For cannabis compartments, these estimates are comparable with those obtained on the basis of the online survey.

\section{The forward and backward transition rates between regular and intensive users}

These transition rates have been estimated with the same approach as those described before on the basis of SPS 2010 and SPS 2011 which is a very conservative approach but allows for an estimate of the order of magnitude of the parameters that can then be adjusted by means of sensitivity analysis and qualitative information from the survey in the communities and lowthreshold services. The initial values of the parameters are: $\gamma_{\mathrm{ri}}=0.16 ; \gamma_{\mathrm{ir}}=0.09 ; \alpha_{\mathrm{ri}}=0.67 ;$ and $\alpha_{\mathrm{ir}}=0.074$.

\section{The forward and backward transition rates between the $C$ and the $A$ compartments and the transition rates between $C$ and $H$ and $A$ and $H$ compartments}

First, it is assumed that the transitions between the $C$ and $A$ compartments for intensive users are negligible. This hypothesis is supported by the results of the 2010 and 2012 surveys in the Communities and Low Threshold Services and of the interviews with these clients. On the basis of the available data, it is possible to estimate the transitions for regular users only. These rates have been estimated using data from the 2010 and 2012 surveys in the Communities and Low Threshold Services that allows for an estimate of the distribution of the lag between only cannabis use and the use of other substances (mainly heroin or cocaine). The average time lag is 2.5 years and the proportion that experiences this transition between compartments is about $10 \%$ (from the literature and the information from SPS 2010 and 2011 and the online survey). Thus, the forward transition rate is $\delta_{\mathrm{rr}}=0.04$. The backward transition rate can be estimated on the basis of the MI data on registration for personal use and results at $\beta_{\mathrm{rr}}=0.005$.

The forward and backward transition rates for the $C$ and $A$ compartments of occasional users cannot be estimated on the basis of the available data. They are estimated on the basis of the known steady state. 
The estimated values of these parameters are: $\beta_{\mathrm{oo}}=0.075 ; \quad \delta_{\mathrm{oo}}=0.08 ; \quad v_{C_{\mathrm{i}}}=0.05 ; \quad v_{A_{\mathrm{i}}}=0.45 ;$ $\mu_{C_{\mathrm{i}}}=0.06$; and $\mu_{A_{\mathrm{i}}}=0.3$.

Using these estimates, it is possible to simulate the model and to adjust the parameters to perform scenario analyses as has been shown in the 'Different scenarios and possible interventions' section.

Declaration of interest: The authors report no conflicts of interest. The authors alone are responsible for the content and writing of the article.

Work developed within the framework of the EU project JUST/2010/DPIP/AG/1410: New methodological tools for policy and programme evaluation.

This publication has been produced with the financial support of the Prevention and Information Programme of the European Commission. The contents of this publication are the sole responsibility of the authors and can in no way be taken to reflect the views of the European Commission.

\section{REFERENCES}

Behrens, D.A., Caulkins, J.P., Tragler, G., \& Feichtinger, G. (2000). Optimal control of drug epidemics: Prevent and treat But not at the same time? Management Science, 46, 333-347.

Behrens, D.A., Caulkins, J.P., Tragler, G., Haunschmied, J.L., \& Feichtinger, G. (1999). A dynamic model of drug initiation: Implications for treatment and drug control. Mathematical Biosciences, 159(1), 1-20.

Billard, L., \& Dayananda, P.W.A. (1993). Drug addictionpusher generated from addicts. Biometrical Journal, 35, 227-244.

Bouchard, M., \& Tremblay, P. (2005). Risks of arrests across drug markets: A capture-recapture analysis of "hidden" dealer and user population. Journal of Drug Issues, 35, 733-754.

Caulkins, J.P., Behrens, D.A., Knoll, C., Tragler, G., \& Zuba, D. (2004). Markov chain modeling of initiation and demand: The case of the U.S. cocaine epidemic. Health Care Management Science, 7, 319-329.

Caulkins, J.P., Dietze, P., \& Ritter, A. (2007). Dynamic compartmental model of trends in Australian drug use. Health Care Management Science, 10, 151-162.

Dipartimento per le Politiche Antidroga. (2010). Relazione annuale al Parlamento 2010 sull'uso di sostanze stupefacenti e sulle tossicodipendenze in Italia, Presidenza del Consiglio dei Ministri. Retrieved from http://www.politicheantidroga.it/ progetti-e-ricerca/relazioni-al-parlamento/relazione-annuale2010/presentazione.aspx

Dipartimento per le Politiche Antidroga. (2011). Relazione annuale al Parlamento 2010 sull'uso di sostanze stupefacenti e sulle tossicodipendenze in Italia, Presidenza del Consiglio dei Ministri. Retrieved from http://www.politicheantidroga.it/ progetti-e-ricerca/relazioni-al-parlamento/relazione-annuale2011/presentazione.aspx

Fabi, F., Mammone, A., \& Rossi, C. (2012). Monitoring and assessing drug policies: Indicators of drug use in the teenage population as a tool for cross country comparisons. Sixth Annual Conference of the International Society for the Study of Drug Policy, Canterbury, UK, 30-31 May 2012.

Homer, J.B. (1993). A system dynamics model for cocaine prevalence estimation and trend projection. Journal of Drug Issues, 23, 251-279.

Levy, D.T., Chaloupka, F., Gitchell, J., Mendez, D., \& Warner, K.E. (2002). The use of simulation models for the surveillance: Justification and understanding of tobacco control policies. Health Care Management Science, 5, 113-120.

Levy, D.T., \& Friend, K. (2002). Examining the effects of tobacco treatment policies on smoking rates and smoking related deaths using the SimSmoke computer simulation model. Tobacco Control, 11, 47-54.

Mackintosh, D.R., \& Stewart, G.T. (1979). A mathematical model of a heroin epidemic: Implications for control policies. Journal of Epidemiology and Community Health, 33, 299-304.

Mascioli, F., \& Rossi, C. (2008). Capture-recapture methods to estimate prevalence indicators for evaluating drug policies. UN Bulletin on Narcotics, LX, 5-25.

Rey, G.M., Rossi, C., \& Zuliani, A. (Eds.). (2011). Il mercato delle droghe: Dimensione, protagonisti, politiche. Venezia: Marsilio editori.

Rossi, C. (2001). A Mover-Stayer type model for problem drug use epidemic. Bulletin on Narcotics, LIII, 39-64.

Rossi, C. (2002). The role of dynamic modelling in drug abuse epidemiology. Bulletin on Narcotics, LIV, 33-44.

Rossi, C. (2004). Operational models for epidemic of problematic drug use: The Mover-Stayer approach to heterogeneity. Socio-Economic Planning Sciences, 38, 73-90.

Scalia Tomba, G., Rossi, C., Taylor, C., Klempova, D., \& Wiessing, L. (2008). Guidelines for estimating the incidence of problem drug use. Lisbon: EMCDDA.

Wiessing, L.G., Hartnoll, R., \& Rossi, C. (2001). The epidemiology of drug use at macro level: Indicators, models and policy-making. Bulletin on Narcotics, LIII, 119-133. 